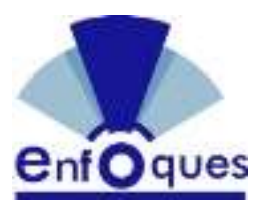

\section{COMPONENTES DE LOS INDICADORES DE GESTIÓN DE LA INDUSTRIA PETROLERA VENEZOLANA}

\author{
COMPONENTS OF THE MANAGEMENT INDICATORS OF THE \\ VENEZUELAN OIL INDUSTRY \\ COMPONENTES DOS INDICADORES DE GESTÃO DA INDÚSTRIA \\ PETROLÍFERA VENEZUELANA
}

\section{Elba García}

Artículo recibido julio 2020 | Arbitrado agosto 2020 | Publicado 01 de octubre 2020

\section{Resumen}

La investigación tuvo como objetivo describir los componentes de los indicadores de gestión que se aplican en la industria petrolera venezolana. El estudio fue descriptivo, con diseño no experimental, transeccional, y de campo. Los datos fueron recolectados de una población finita, conformada por gerentes, líderes, supervisor mayor y planificadores de las gerencias pertenecientes a la División Sur Lago-Trujillo de la industria petrolera venezolana. Para la recolección de datos se aplicó la técnica de la encuesta, con un cuestionario contentivo de 18 ítems bajo una escala dicotómica. La validación del instrumento se realizó mediante el juicio expertos, y para calcular su confiabilidad se empleó el coeficiente Küder-Richardson, donde se obtuvo como valor 0,872 . El análisis de los datos se realizó mediante estadística descriptiva, bajo el estudio de las frecuencias relativas. Se evidenció alta aplicación de todos los componentes medidos: definición, objetivo, valor de referencia, responsabilidad, puntos de medición y periodicidad.
\end{abstract}

Palabras clave: Componentes, definición, indicadores de gestión, objetivo, periodicidad, puntos de medición, responsabilidad, valor de referencia

\begin{abstract}
The research aimed to describe the components of the management indicators that are applied in the Venezuelan oil industry. The study was descriptive, with a non-experimental, transectional, and field design. The data was collected from a finite population, made up of managers, leaders, senior supervisor and planners of the managers belonging to the South Lake-Trujillo Division of the Venezuelan oil industry. For the data collection, the survey technique was applied, with a questionnaire containing 18 items under a dichotomous scale. The instrument was validated using expert judgment, and the Küder-Richardson coefficient was used to calculate its reliability, where it was obtained as a value of 0.872 . Data analysis was performed using descriptive statistics, under the study of relative frequencies. High application of all the measured components was evident: definition, objective, reference value, responsibility, measurement points and periodicity.
\end{abstract}

Key words: Components, definition, management indicators, objective, periodicity, measurement points, responsibility, reference value

\section{Elba García}

elbagarcia33@gmail.com

Orcid: 0000-0002-9558-0145

Universidad del Zulia. Núcleo Costa Oriental del Lago, Venezuela.

Licenciada en Contaduría, egresada de la Universidad del Zulia. Núcleo Costa Oriental del Lago. Magíster Scientiarum en Gerencia de Empresas. Mención: Gerencia de Operaciones. Universidad del Zulia, Núcleo Costa Oriental del lago. Venezuela.

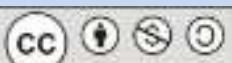

Revista Enfoques 


\section{Resumo}

O objetivo da pesquisa foi descrever os componentes dos indicadores de gestão aplicados na indústria do petróleo venezuelana. O estudo foi descritivo, com desenho não experimental, transversal e de campo. Os dados foram coletados de uma população finita, composta por gerentes, líderes, supervisor sênior e planejadores das administraçôes pertencentes à Divisão Lago Sul-Trujillo da indústria petrolífera venezuelana. Para a coleta de dados, foi aplicada a técnica de survey, com questionário contendo 18 itens em escala docotômica. A validação do instrumento foi realizada por meio de julgamento de especialistas, e o coeficiente de KüderRichardson foi utilizado para calcular sua confiabilidade, onde foi obtido o valor de 0,872 . A análise dos dados foi realizada por meio de estadística descritiva, sob o estudo de frequências relativas. Foi evidenciada alta aplicação de todos os componentes medidos: definição, objetivo, valor de refêrencia, responsabilidade, pontos de medição e periodicidade.

Palavras-chave: Componentes, definição, indicadores de gestção, objetivo, periodicidade, pontos, de medição, responsabilidade, valor de refêrencia

\section{INTRODUCCIÓN}

A medida que la gerencia detecte la necesidad de mejorar sus resultados operacionales y financieros, deberán basarse en el diseño de técnicas innovadoras que le permitan obtener mejoras progresivas a corto, mediano y largo plazo, mediante estrategias que conlleven al cumplimiento de todos los objetivos, desde el más general hasta los específicos, controlando cada una de estas técnicas a través de indicadores que permitan el seguimiento al desempeño organizacional. De manera que, el uso de indicadores en la auditoría de la empresa, obedece a la necesidad de disponer de un marco de referencia para evaluar los resultados de la gestión de la organización.

Bajo esta perspectiva, los indicadores de gestión aportan elementos sustanciales para promover el mejoramiento continuo de los procesos, productos y servicios; así como el empleo puntual de sus recursos, a la vez que abre la posibilidad de instrumentar los cambios necesarios, para lograr el efectivo cumplimiento de su objetivo.

En este sentido, para Calzón (2012), los indicadores son instrumentos básicos de las prácticas directas para el control y despliegue de las estrategias, que sirven de mecanismo idóneo para garantizar el despliegue de políticas corporativas y acompañar el desenvolvimiento de los planes; es una necesidad en la medida en que crece la complejidad de la organización, lo cual demanda descentralización y flexibilidad.

De esta manera, en el contexto empresarial surge la concepción del sistema de medición de gestión, basado en el uso de indicadores como herramienta competitiva que soporta a las empresas para optimizar sus recursos generando valor en sus procesos, cumpliendo con las entregas al consumidor final con altos niveles de satisfacción (Serna, 2008). Para ello las empresas deben tener claro lo que implica la medición de su gestión. La primera establece un plano operativo de la acción (control), mientras que la segunda es el proceso gerencial estratégico (gestión).

En este marco de referencia, para Serna (2008), el sistema de medición de gestión se estructura de un conjunto de indicadores medibles, derivados del plan estratégico, que permiten evaluar con índices el alineamiento entre estrategias, objetivos, acciones y resultados, por tanto, estos indicadores permiten determinar el desempeño de la organización frente a su direccionamiento estratégico.

En concordancia con lo expuesto, Venezuela no escapa de las exigencias planteadas a nivel mundial para el crecimiento o fortalecimiento de sus organizaciones, donde 
las industrias puedan ser capaces de sobrepasar las expectativas del cliente, brindando calidad y diversidad, del mismo modo poseer la capacidad productiva y organizacional para hacer intercambios comerciales con el mercado extranjero.

Atendiendo a esto, se hace necesario un estudio más profundo de la situación de las industrias, a fin de determinar la existencia o modos de implantación de los indicadores de gestión, tanto en lo estratégico como en lo operativo, con el fin de poder realizar un máximo aprovechamiento de las oportunidades que le brinda el entorno así como de las fortalezas que las industrias posean.

Bajo tales consideraciones, el uso adecuado de indicadores de gestión representa una función clave en el apoyo a sus operaciones, propiamente dichas, y contribuir al logro de los objetivos de la organización. Así, estas organizaciones han concentrado esfuerzos en el aumento de sus capacidades, evolucionando hacia la mejora de su eficiencia, llevando a las mismas a la obtención de sus metas. En esta perspectiva, al sector petrolero a nivel mundial se le confiere el carácter de base energética clave de la sociedad moderna, con base en el petróleo como fuente de energía con múltiple utilización, por ello la industria petrolera es considerada una de las más importantes.

En este contexto, la industria petrolera en Venezuela no debería quedar relegada del resto del mundo. En cuanto al caso específico de estudio, se reseña que PDVSA dentro de su estructura organizacional se ubican las divisiones de la región occidente (Costa Oriental, Lago, Sur Lago-Trujillo y Costa Occidental), encargadas de establecer planes estratégicos para contribuir en la ejecución a nivel operacional de las actividades de perforación, rehabilitación y servicios a pozos; procesos que generan con frecuencia mensual informes de indicadores denominado rendición de cuentas, permitiendo el análisis de la ejecución real en comparación con la planificada del año fiscal.

Estas divisiones emplean un proceso de medición de gestión normalizado, basado en las mejores prácticas, guiadas por normas y procedimientos creadas por ellas mismas, las cuales contienen lineamientos prácticos para la efectiva ejecución de la medición y control de su gestión de manera sistemática u ordenada, tratando de no omitir ningún paso importante. Garantizando de esta manera un alto grado de confianza en el éxito y en el cumplimiento de los requisitos solicitados por PDVSA. Éste es el enfoque de las divisiones de la región occidente, utilizado a fin de alcanzar las más altas metas de eficiencia, en un mundo totalmente cambiante como el actual.

Ahora bien, se han seleccionado como ámbito de análisis las gerencias de la División Sur Lago-Trujillo (Operaciones de perforación, Mantenimiento a Taladro, Rehabilitación de Pozos, LSYM y Operaciones de Producción), en las cuales se ha venido observando un mayor reconocimiento, por parte del estrato gerencial, de la importancia que tiene lograr una gestión efectiva para alcanzar un control eficiente de sus procesos.

A partir de los aspectos mencionados, se hizo notoria la necesidad de describir los indicadores de gestión que se aplican en la industria petrolera venezolana, a fin de ofrecer elementos de relevancia para las gerencias de la División Sur Lago-Trujillo de la industria petrolera venezolana, específicamente en cuanto a sus componentes para así tomar decisiones a favor de la consecución de los objetivos organizacionales definidos.

\section{MÉTODO}

El tipo de investigación que se aplicó en este trabajo, de acuerdo a la información que se poseía y nivel de análisis que se hizo, es un 
estudio de carácter descriptivo, con diseño no experimental, transeccional, y de campo. Los datos fueron recolectados de una población finita, conformada por gerentes, líderes, supervisor mayor y planificadores de las gerencias pertenecientes a la División Sur LagoTrujillo de la industria petrolera venezolana.

Para la recolección de datos se aplicó la técnica de la encuesta, con un cuestionario contentivo de 18 ítems bajo una escala dicotómica, de dos (2) alternativas de respuesta, representada de la siguiente manera: Si o No. La validación del instrumento se realizó mediante el juicio expertos, y para calcular su confiabilidad se empleó el coeficiente Küder-Richardson, donde se obtuvo como valor 0,872 al incluir todos los ítems, la cual es considerada de muy alta confiabilidad, confirmándose así un instrumento que cumplía con las dos condiciones básicas ser válido y confiable.

El análisis de los datos se realizó mediante estadística descriptiva, bajo el estudio de las frecuencias relativas. Para tal efecto, la investigadora diseñó un (1) baremo, para el análisis e interpretación de las frecuencias de los ítems, indicadores, dimensiones, en términos de medir el nivel de aplicación de cada uno de ellos en la gestión, mostrado en la tabla 1.

Tabla 1. Baremo de interpretación de la frecuencia

\begin{tabular}{cc}
\hline & RANGO PARA FRECUENCIA RELATIVA \\
\hline $\mathrm{SI} \geq 70 \%$ & Alta aplicación / Alta fortaleza \\
$40 \% \leq \mathrm{SI}<70 \%$ & Mediana aplicación / Leve fortaleza \\
$\mathrm{SI}<40 \%$ & Baja aplicación / Debilidad \\
\hline
\end{tabular}

\section{RESULTADOS}

En la tabla 2 se muestran los resultados para el indicador definición, de la dimensión denominada componentes de los indicadores de gestión. De acuerdo al baremo establecido, en las gerencias bajo estudio, se obtuvo alta aplicación de la definición como parte de los componentes de los indicadores de gestión que deben poseer dichos indicadores, situación que se devela al ubicarse en promedio el $100 \%$ de respuestas en la opción sí, confiriéndole una alta fortaleza a estas gerencias. 
Tabla 2. Indicador: Definición

\section{En la gerencia donde usted labora:}

1. ¿En la definición de los indicadores de gestión se señalan metas que se convierten en referencias para el control de dicha gestión?

2. ¿Los indicadores de gestión que se utilizan producen información necesaria para analizar el desempeño de cualquier área de la organización?

3. ¿Los indicadores de gestión implementados permiten verificar el cumplimiento de los objetivos en términos de resultados?

\begin{tabular}{|c|c|c|c|c|c|c|c|c|c|c|c|c|}
\hline \multicolumn{8}{|c|}{ ITEMS } & \multicolumn{5}{|c|}{ INDICADOR } \\
\hline $\mathbf{N}^{\circ}$ & Si & $\%$ & No & $\%$ & $\mathbf{n}$ & \multicolumn{2}{|c|}{ Categoría } & Si & $\%$ & No & $\%$ & $\mathbf{n}$ \\
\hline 1 & 38 & 100 & 0 & 0,00 & 38 & Alta & AF & 38 & 100 & 0 & 0 & 38 \\
\hline 2 & 38 & 100 & 0 & 0,00 & 38 & Alta & $\mathrm{AF}$ & \multicolumn{5}{|c|}{ CATEGORÍA } \\
\hline 3 & 38 & 100 & 0 & 0,00 & 38 & Alta & $\mathrm{AF}$ & \multicolumn{5}{|c|}{ Alta aplicación / Alta fortaleza } \\
\hline
\end{tabular}

Estos resultados reflejan que, según los encuestados, en estas gerencias se da alta aplicación a los procesos vinculados a la definición de los indicadores, en cuanto a si en la definición de los indicadores de gestión se señalan metas que se convierten en referencias para el control de dicha gestión (100\%), los indicadores de gestión que se utilizan producen información necesaria para analizar el desempeño de cualquier área de la organización (100\%), y si los indicadores de gestión implementados permiten verificar el cumplimiento de objetivos en términos de resultados (86,67\%); constituyendo esas acciones en altas fortalezas de la medición de su gestión.

Dado los resultados mostrados, se evidencia alta congruencia con lo planteado en la teoría por Fernández (2012), para quien la definición es la descripción del resultado que se pretende obtener mediante la ejecución de un proceso. Produce información para analizar el desempeño de cualquier área de la organización y verificar el cumplimiento de los objetivos en términos de resultados.

También establecen alta coincidencia con lo planteado por investigadora cuando asegura que, la definición representa la característica principal dentro de las actividades de una organización para el logro de los objetivos en términos de resultados. Este componente clave de la construcción de indicadores, debe cumplir con los requisitos para lo cual fue creado, es decir, ser una expresión que cuantifique el estado de la característica o hecho que se desea controlar.

En la tabla 3, se muestran los valores obtenidos de las respuestas correspondientes al indicador objetivo. Se observa como el $100 \%$ de los sujetos encuestados respondieron de manera afirmativa a las proposiciones emitidas, indicando que las actividades relacionadas a los ítems tienen alta aplicación en la medición de gestión que ejecutan las gerencias analizadas, siendo esto una alta fortaleza para la mismas, de acuerdo al baremo utilizado para tal fin. 
Tabla 3. Indicador: Objetivo

En la gerencia donde usted labora:

4. ¿ ¿El indicador de gestión aplicado persigue el logro de un objetivo establecido, como por ejemplo el mejoramiento que se busca?

5. ¿El indicador de gestión seleccionado permite establecer el sentido de las mejoras que se requieren?

6. ¿Los indicadores de gestión aplicados permiten seleccionar acciones preventivas así como correctivas en una sola dirección?

\begin{tabular}{|c|c|c|c|c|c|c|c|c|c|c|c|c|}
\hline \multicolumn{8}{|c|}{ ITEMS } & \multicolumn{5}{|c|}{ INDICADOR } \\
\hline $\mathbf{N}^{\circ}$ & Si & $\%$ & No & $\%$ & $\mathbf{n}$ & \multicolumn{2}{|c|}{ Categoría } & Si & $\%$ & No & $\%$ & $\mathbf{n}$ \\
\hline 4 & 38 & 100 & 0 & 0,00 & 38 & Alta & $\mathrm{AF}$ & 38 & 100 & 0 & 0 & 38 \\
\hline 5 & 38 & 100 & 0 & 0,00 & 38 & Alta & $\mathrm{AF}$ & \multicolumn{5}{|c|}{ CATEGORÍA } \\
\hline 6 & 38 & 100 & 0 & 0,00 & 38 & Alta & $\mathrm{AF}$ & \multicolumn{5}{|c|}{ Alta aplicación / Alta fortaleza } \\
\hline
\end{tabular}

Al detallar estos resultados se aprecia que, para el $100 \%$ de los encuestados se da alta aplicación a las siguientes actividades: el indicador de gestión aplicado persigue el logro de un objetivo establecido, como por ejemplo el mejoramiento que se busca; asimismo, el indicador de gestión seleccionado permite establecer el sentido de las mejoras que se requieren; y los indicadores de gestión aplicados permiten seleccionar acciones preventivas así como correctivas en una sola dirección; constituyéndolas en altas fortalezas de la medición de gestión que llevan a cabo.

Los resultados validan, en alta medida, lo expuesto en la teoría que soporta la investigación, como es el caso de Mora (2011), para quien el objetivo es lo que persigue el indicador seleccionado; indica el mejoramiento que se busca y el sentido de esa mejora (maximizar, minimizar, eliminar, mejorar, entre otros), en consecuencia, permite seleccionar y combinar acciones preventivas y correctivas en una sola dirección. Esta combinación dependerá de la magnitud de los problemas y el momento (oportunidad) de intervención.

También validan, de forma alta, lo afirmado por la investigadora para quien, el objetivo del indicador de gestión, como componente básico de su construcción, debe dejar claro el mejoramiento que se busca y el sentido de esa mejora permitiendo seleccionar y combinar las acciones a seguir para garantizar la mejora en los procesos.

La tabla 4, muestra las respuestas correspondientes al indicador valor de referencia, en la cual se aprecia cómo el $100 \%$ de los encuestados, en las gerencias bajo estudio, considera con alta aplicación las actividades relacionadas al indicador, asignándole una alta fortaleza al respecto. Esta situación es explicada por el comportamiento de las respuestas afirmativas dadas a cada ítem involucrado, las cuales arriban a la categoría de alta aplicación.

De tal forma, para el $100 \%$ de los encuestados, las gerencias estudiadas cuentan con un nivel de referencia para comparar el valor de los indicadores de gestión aplicados; además, utilizan valores de referencia para la medición de los indicadores de gestión, permitiéndoles medir como ha sido la tendencia en el transcurso del tiempo. Así como el valor de referencia utilizado les permite conocer si el proceso está o ha estado controlado; siendo altas fortalezas en estos aspectos de su medición de gestión. 
Los resultados concuerdan con lo establecido por Fernández (2012), para quien el valor de referencia muestra cómo ha sido la tendencia en el transcurso del tiempo, permite proyectar y calcular valores esperados en el período. Señala la variación de resultados, su capacidad real, actual y probada, informa si el proceso está, o ha estado, controlado. El valor de referencia dice lo que se ha hecho, pero no dice el potencial alcanzable.

Tabla 4. Indicador: Valor de referencia

\section{En la gerencia donde usted labora:}

7. ¿Cuentan con un nivel de referencia para comparar el valor de los indicadores de gestión aplicados?

8. ¿Utilizan valores de referencia para la medición de los indicadores de gestión, permitiéndoles medir como ha sido la tendencia en el transcurso del tiempo?

9. ¿El valor de referencia utilizado les permite conocer si el proceso está o ha estado controlado?

\begin{tabular}{|c|c|c|c|c|c|c|c|c|c|c|c|c|}
\hline \multicolumn{8}{|c|}{ ITEMS } & \multicolumn{5}{|c|}{ INDICADOR } \\
\hline $\mathbf{N}^{\circ}$ & Si & $\%$ & No & $\%$ & $\mathbf{n}$ & \multicolumn{2}{|c|}{ Categoría } & Si & $\%$ & No & $\%$ & $\mathbf{n}$ \\
\hline 7 & 38 & 100 & 0 & 0,00 & 38 & Alta & $\mathrm{AF}$ & 38 & 100 & 0 & 0 & 38 \\
\hline 8 & 38 & 100 & 0 & 0,00 & 38 & Alta & $\mathrm{AF}$ & \multicolumn{5}{|c|}{ CATEGORÍA } \\
\hline 9 & 38 & 100 & 0 & 0,00 & 38 & Alta & $A F$ & \multicolumn{5}{|c|}{ Alta aplicación / Alta fortaleza } \\
\hline
\end{tabular}

Asimismo, validan lo considerado por la investigadora cuando afirma que, el valor de referencia, como componente básico del indicador de gestión, es un parámetro establecido para medir el valor alcanzado por el indicador, el cual permite medir y comparar el comportamiento de los resultados obtenidos durante un determinado proceso de gestión.

De seguido, en la tabla 5 se muestran los resultados para el indicador responsabilidad, el cual fue considerado para medir la dimensión componentes de los indicadores de gestión. Como se evidencia, el $82 \%$ de las respuestas se posicionaron en la opción afirmativa, otorgándole a este indicador la categoría de alta aplicación, indicándose como una alta fortaleza de la medición de gestión llevada a cabo.
Al detalle se visualiza como todos los ítems arriban a la categoría de alta aplicación, por ende son altas fortalezas de la medición de su gestión. Así entonces, otorgan alta aplicación en cuanto a sí: han especificado claramente a quienes les corresponde actuar en cada momento, para cada nivel de la organización, frente a la información que les está suministrando el indicador de gestión (94,74\%); tienen claramente establecida la responsabilidad de acción frente a la desviación de los resultados respecto a las referencias escogidas en cada uno de los procesos $(81,58 \%)$; y tienen claramente establecida la responsabilidad de acción frente a la información que suministra el indicador (a quien le corresponde tomar acciones correctivas o preventivas) $(71,05 \%)$. 
Tabla 5. Indicador: Responsabilidad

\section{En la gerencia donde usted labora:}

10. ¿Tienen claramente establecida la responsabilidad de acción frente a la información que suministra el indicador (a quien le corresponde tomar acciones correctivas o preventivas)?

11. ¿Tienen claramente establecida la responsabilidad de acción frente a la desviación de los resultados respecto a las referencias escogidas en cada uno de los procesos?

12. ¿Han especificado claramente a quienes les corresponde actuar en cada momento, para cada nivel de la organización, frente a la información que les está suministrando el indicador de gestión?

\begin{tabular}{|c|c|c|c|c|c|c|c|c|c|c|c|c|}
\hline \multicolumn{8}{|c|}{ ITEMS } & \multicolumn{5}{|c|}{ INDICADOR } \\
\hline $\mathbf{N}^{\circ}$ & Si & $\%$ & No & $\%$ & $\mathbf{n}$ & \multicolumn{2}{|c|}{ Categoría } & Si & $\%$ & No & $\%$ & $\mathbf{n}$ \\
\hline 10 & 27 & 71,05 & 11 & 28,95 & 38 & Alta & $A F$ & 31 & 82 & 7 & 18 & 38 \\
\hline 11 & 31 & 81,58 & 7 & 18,42 & 38 & Alta & $\mathrm{AF}$ & \multicolumn{5}{|c|}{ CATEGORÍA } \\
\hline 12 & 36 & 94,74 & 2 & 5,26 & 38 & Alta & $\mathrm{AF}$ & \multicolumn{5}{|c|}{ Alta aplicación / Alta fortaleza } \\
\hline
\end{tabular}

Estos resultados logran validar la teoría expuesta por Corredor (2007), quien lo define como el componente del indicador que permite establecer de manera clara el modo de actuar frente a la información que suministra el indicador y su posible desviación respecto a las referencias escogidas. También alcanzan congruencia con lo expresado por la investigadora, cuando asume que los indicadores se deben desarrollar a lo largo de la organización, en todos sus niveles, y dado que se asocian a los resultados sobre productos/servicios de cada nivel, se incluyen entonces los directivos o líderes a cargo que son responsables por ellos. Por lo que, el paso siguiente a la identificación de lo que se medirá es establecer las responsabilidades institucionales para el cumplimiento en el manejo de la información, tanto para alimentar el indicador como para su análisis y presentación de resultados.

La tabla 6, muestra las respuestas correspondientes al indicador puntos de medición. Se aprecia que el $78,95 \%$ de los encuestados consideraron que, en las gerencias bajo estudio, se da alta aplicación a las actividades o procesos relacionados al indicador. De tal forma, se otorga alta aplicación a todos los ítems medidos, donde el $78,95 \%$ de los encuestados afirma que: tienen claramente definido el momento donde deben hacerse las mediciones de los indicadores; asimismo, los medios con los cuales hacer las mediciones de los indicadores; y quiénes hacen las lecturas de las mediciones; viendo estos aspectos como altas fortalezas de la medición de gestión que ejecutan.

Así, se logra congruencia con lo postulado por Mora (2011), para quien los puntos de medición establecen la forma cómo se obtienen y conforman los datos, sitios y momento donde deben hacerse las mediciones, los medios con los cuales hacer las medidas, quiénes hacen las lecturas y cuál es el procedimiento de obtención de las muestras, ello permite establecer con claridad la manera de obtener precisión, oportunidad y confiabilidad en las medidas. 
Tabla 6. Indicador: Puntos de medición

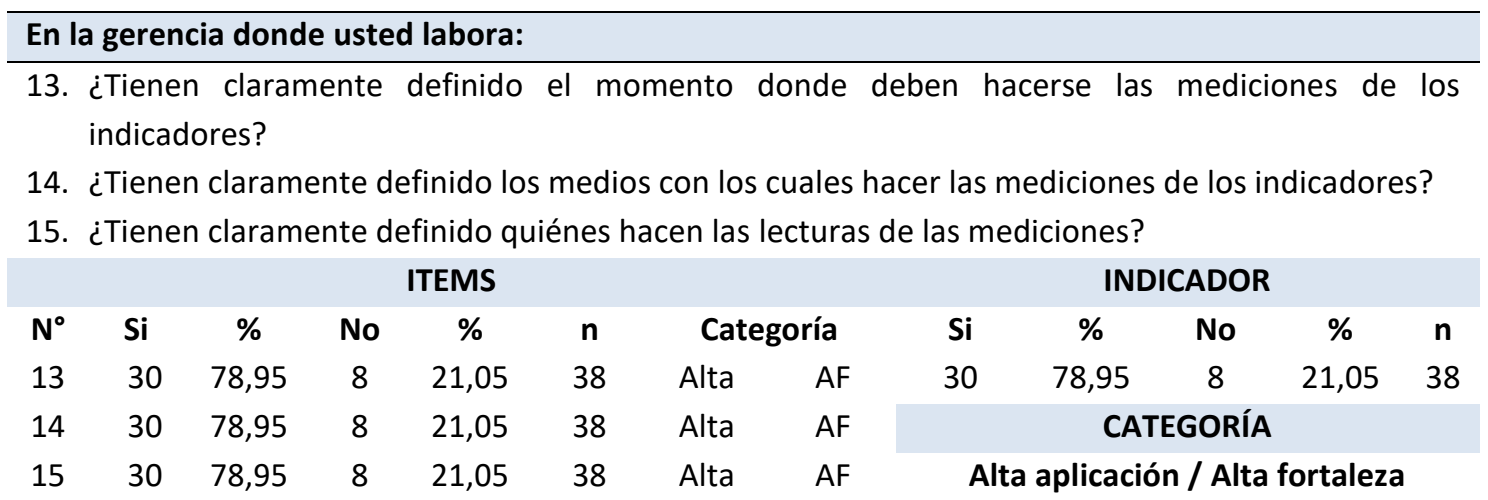

Asimismo, son congruentes con la posición de la investigadora, para quien el punto de medición permite establecer con mayor precisión la confiabilidad de las medidas, puesto que clarifica el cómo se obtendrán y conformaran los datos, momentos en que deberán hacerse las mediciones, medios disponibles para hacer las medidas, quiénes harán las lecturas y cuál será el procedimiento de obtención de las muestras.

Seguidamente, se presentan en la tabla 7 los resultados para el último indicador de la dimensión componentes de los indicadores de gestión, denominado periodicidad. Se observa una concentración de respuestas afirmativas del $89 \%$, otorgándole la categoría de alta aplicación, según el baremo utilizado, indicando una alta fortaleza para la medición de gestión que realizan.
De tal forma, al consultarle si el sistema de medición de la gestión es lo suficientemente ágil o rápido para asegurar la retroalimentación adecuada a cada nivel de la organización donde sean utilizados los indicadores, y si realizan la revisión de los indicadores en correspondencia con la naturaleza del proceso que se quiere medir el 92,11\% respondieron de manera afirmativa, designando alta aplicación a estas actividades, por ende, son altas fortalezas de la gestión. De manera similar, el 81,58\% catalogó de alta aplicación el que se establezca cada cuanto tiempo se tomarán los datos para realizar la medición del desempeño de la gestión, siendo también una alta fortaleza de la medición de su gestión. 
Tabla 7. Indicador: Periodicidad

\section{En la gerencia donde usted labora:}

16. ¿Establecen cada cuanto tiempo se tomarán los datos para realizar la medición del desempeño de la gestión?

17. ¿El sistema de medición de la gestión es lo suficientemente ágil o rápido para asegurar la retroalimentación adecuada a cada nivel de la organización donde sean utilizados los indicadores?

18. ¿Realizan la revisión de los indicadores en correspondencia con la naturaleza del proceso que se quiere medir?

\begin{tabular}{ccccccccccccc}
\multicolumn{1}{c}{ ITEMS } & \multicolumn{1}{c}{ INDICADOR } \\
$\mathbf{N}^{\circ}$ & $\mathbf{S i}$ & $\%$ & No & $\%$ & $\mathbf{n}$ & Categoría & Si & $\%$ & No & $\%$ & $\mathbf{n}$ \\
16 & 31 & 81,58 & 7 & 18,42 & 38 & Alta & AF & 34 & 89 & 4 & 11 & 38 \\
17 & 35 & 92,11 & 3 & 7,89 & 38 & Alta & AF & & \multicolumn{3}{c}{ CATEGORÍA } \\
18 & 35 & 92,11 & 3 & 7,89 & 38 & Alta & AF & \multicolumn{3}{c}{ Alta aplicación / Alta fortaleza } \\
\hline
\end{tabular}

De acuerdo a los resultados, se logra congruencia con Hernández (2012), cuando explica que, la periodicidad debe responder a la interrogante de ¿cuándo hacer la revisión? La respuesta debe estar en correspondencia con la naturaleza del proceso que se quiere medir, a fin de establecer cada cuanto tiempo se tomarán los datos y presentarla en los gráficos de control para estudiar la existencia de tendencias en el desarrollo del proceso. Además, estudiar las causas comunes y especiales, las cuales deberán ser presentadas adecuadamente para facilitar la toma de decisiones, por lo que este sistema debe ser suficientemente ágil y rápido para asegurar retroalimentación adecuada a cada nivel de la organización donde sea utilizado.

También se visualiza coincidencia con la investigadora, para quien la periodicidad, como componente básico de la construcción de indicadores de gestión, es el período de la medida del proceso, estableciendo el tiempo de toma de datos y su presentación en gráficos. Se refiere a la frecuencia con que la que se obtiene la información sea esta anual, semestral, mensual, entre otros.
Para la dimensión componentes de los indicadores de gestión, cuyos resultados se concentran en la tabla 8 , se observa cómo el $91 \%$, en promedio, de los encuestados consideraron la opción afirmativa, indicando alta aplicación de los componentes de los indicadores, dentro de la medición de gestión que realizan las gerencias de la División Sur Lago-Trujillo de la industria petrolera venezolana, delineando este aspecto como alta fortaleza de su medición de gestión.

Al detalle, se aprecia que todos los componentes de los indicadores de gestión fueron catalogados con alta aplicación: definición (100\%), objetivo (100\%), valor de referencia (98\%), responsabilidad (82\%), puntos de medición (79\%), y periodicidad (89\%); por tanto, las delinean como altas fortalezas de la medición de gestión que realizan estas gerencias.

Estos resultados validan los postulados de Mora (2011) y Corredor (2007), para quienes al construir un indicador, se deben considerar los siguientes componentes: definición, objetivo, valor de referencia, responsabilidad, puntos de medición y la periodicidad. 
Tabla 8. Dimensión: Componentes de los indicadores de gestión

\begin{tabular}{lccccc}
\hline \multicolumn{1}{c}{ INDICADOR } & Si & \% & No & \% & BAREMO \\
\hline Definición & 38 & 100 & 0 & 0 & Alta aplicación / Alta fortaleza \\
Objetivo & 38 & 100 & 0 & 0 & Alta aplicación / Alta fortaleza \\
Valor de referencia & 37 & 98 & 1 & 2 & Alta aplicación / Alta fortaleza \\
Responsabilidad & 31 & 82 & 7 & 18 & Alta aplicación / Alta fortaleza \\
Puntos de medición & 30 & 79 & 8 & 21 & Alta aplicación / Alta fortaleza \\
Periodicidad & 34 & 89 & 4 & 11 & Alta aplicación / Alta fortaleza \\
\multicolumn{1}{c}{ Totales } & $\mathbf{3 5}$ & $\mathbf{9 1}$ & $\mathbf{3}$ & $\mathbf{9}$ & Alta aplicación / Alta fortaleza \\
\hline
\end{tabular}

Fuente: Elaboración propia (2016)

También alcanzan congruencia con lo expresado por la investigadora, cuando afirma que todos los componentes que deben considerarse en la construcción de los indicadores de gestión deben estar enfocados al logro de las condiciones básicas, asegurando el monitoreo de la gestión, así como el que las actividades vayan en el sentido correcto, permitiendo evaluar los resultados de la gestión frente a sus objetivos, metas $y$ responsabilidades, y facilitando la toma de decisiones; siendo estos: definición, objetivo, valor de referencia, responsabilidad, puntos de medición y periodicidad.

\section{CONCLUSIONES}

En cuanto al objetivo, donde se describieron los componentes de los indicadores de gestión que se aplican en la industria petrolera venezolana, se encontró que estos componentes ostentan alta aplicación en el sistema de medición de la gestión que desarrollan las gerencias bajo estudio. En específico, se evidenció alta aplicación de todos los componentes medidos: definición, objetivo, valor de referencia, responsabilidad, puntos de medición y periodicidad; por tanto, las delinean como altas fortalezas de la medición de gestión que se realiza.

Sin embargo, se acota que en el componente responsabilidad se detectó una actividad cuya concentración de respuesta está muy cercana al piso del baremo para la categoría alta aplicación ( $\mathrm{SI} \geq 70 \%$ ), medida por el ítem 10, referida a si tienen claramente establecida la responsabilidad de acción frente a la información que suministra el indicador (a quien le corresponde tomar acciones correctivas o preventivas), lo cual puede ser considerado como una oportunidad de mejora.

\section{REFERENCIAS}

Calzón, F. (2012). Competitividad en el mercado. Introducción a la economía empresarial. Editorial ESIC. Madrid. España

Corredor, J. (2007). La planificación estratégica. Vadell Hermanos Editores. Venezuela

Fernández, E. (2012) Indicadores de gestión empresarial. Editorial Asociación Española para la Calidad. Editorial EUMED. Universidad de Málaga. España

Hernández, G. (2012). Guía práctica de indicadores de gestión para establecimientos. Editorial ACARPANTE. México

Mora, A. (2011). Indicadores de gestión. Tercera edición. Editorial Eco ediciones. Bogotá. Colombia

Serna, H. (2008). Gerencia Estratégica. Teoría, Metodología. Alineamiento, Implementación y Mapas Estratégicos, Índices de Gestión. Décima edición. 3R Editores. Bogotá, Colombia 